\title{
FEEDBACK, TRACE AND FIXED-POINT SEMANTICS
}

\author{
P. Katis ${ }^{1}$, Nicoletta SABAdini ${ }^{1}$ And \\ Robert F.C. WALters ${ }^{1,2}$
}

\begin{abstract}
We introduce a notion of category with feedback-withdelay, closely related to the notion of traced monoidal category, and show that the Circ construction of [15] is the free category with feedback on a symmetric monoidal category. Combining with the Int construction of Joyal et al. [12] we obtain a description of the free compact closed category on a symmetric monoidal category. We thus obtain a categorical analogue of the classical localization of a ring with respect to a multiplicative subset. In this context we define a notion of fixedpoint semantics of a category with feedback which is seen to include a variety of classical semantics in computer science.
\end{abstract}

Mathematics Subject Classification. 68Q55, 68Q70, 18D10.

\section{INTRODUCTION}

There has recently been an increasing interest in algebras of systems (or behaviours) in which there is a feedback or fixed-point operation. One source of this work is Kleene's theory of regular languages, the algebra of Conway [10], and process algebras. Another starting point is the work of Elgot $[11,12]$ on singlesorted algebraic theories with a fixed point operation, developed by Bloom and Ésik, many of their results on the equational properties of fixed points being summarized in the book [5]. There are also the network algebras of Stefanescu [34] with the emphasis on feedback.

What has emerged recently are connections with other areas of algebra and geometry. It has become clear that the basic algebra involved is that of monoidal categories $\mathbf{C}$, either symmetric or braided [16]; the principal operations are tensor and composition. Our concern in this paper is with symmetric, even strict,

\footnotetext{
1 Dipartimento di Scienze CC. FF.MM.,Università degli Studi dell'Insubria, Como, Italy; e-mail: nicoletta.sabadini@uninsubria.it, robert.walters@uninsubria.it

2 School of Mathematics and Statistics, University of Sydney, NSW 2006, Australia.
} 
monoidal categories - for this paper we shall refer to them simply as monoidal categories. Similarly when we speak of rings or monoids we will in each case intend commutative. Geometrically, following [27], arrows from a tensor of $m$ objects to a tensor of $n$ objects in a monoidal category may be represented by circuit components with $m$ input wires and $n$ output wires; then the operations become series and parallel operations of components; the symmetry is a twist of the wires. Fixed-point or feedback involves an operation which joins an input wire to an output wire of a single component - however it is not quite clear what operation to take as primitive. In Elgot-Bloom-Ésik the tensor product was taken to be a product (or dually a sum), with associated diagonal "splitting" (or codiagonal "merging") operations on wires. For them fixed-point is an operation fix $_{B}: \mathbf{C}(A \times B, B) \rightarrow \mathbf{C}(A, B)$, geometrically splitting the output line $B$ and joining the second of the $B$ output wires to the $B$ input wire. The fundamental paper of Joyal et al. [15] introduced an axiomatization of a different operation on a monoidal category which they called a trace. Trace is an operation trace $_{U}: \mathbf{C}(A \otimes U, B \otimes U) \rightarrow \mathbf{C}(A, B)$, and geometrically joins the output wire $U$ to the input wire $U$. They show that a traced monoidal category $\mathbf{C}$ may be embedded monoidally in a compact closed category $\operatorname{Int}(\mathbf{C})$. Each object $A$ in a compact closed category $[23,24]$, has an adjoint $A^{\circ}$, with unit $\eta: I \rightarrow A^{\circ} \otimes A$ and counit $\varepsilon: A \otimes A^{o} \rightarrow I$ which may be represented geometrically as curved wires, with shapes $\subset$ and $\supset$ respectively. (Note: wires may be oriented, the identity on $A^{\circ}$ being a wire from right to left.) In terms of the unit and counit fix and trace may then be expressed as follows:

$$
\begin{aligned}
& \operatorname{trace}_{U}(\alpha: A \otimes U \rightarrow B \otimes U)=\left(1_{B} \otimes \varepsilon_{U}\right)\left(\alpha \otimes 1_{U^{o}}\right)\left(1_{A} \otimes \eta_{U^{o}}\right), \\
& \quad f i x_{B}(\alpha: A \times B \rightarrow B)=\left(1_{B} \times \varepsilon_{B}\right)\left(\Delta_{B} \times 1_{B^{0}}\right)\left(\alpha \times 1_{B^{\circ}}\right)\left(1_{A} \times \eta_{B^{\circ}}\right),
\end{aligned}
$$

both formulae corresponding exactly to the respective geometries:

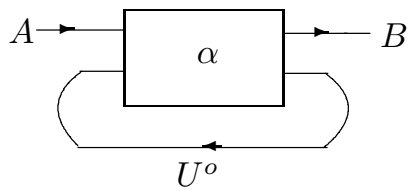

trace $_{U} \alpha$

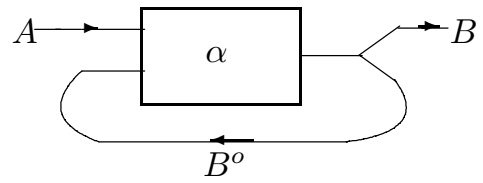

$f i x_{B} \alpha$

A precise connection between the equational theory of fixed points and the notion of trace was made by Hyland and Hasegawa [14] who proved that a traced monoidal category in which the tensor is a product is the same as a Conway theory. In other language this result was already known to Bloom and Ésik [3,4]. It is clear geometrically that if the tensor is either a product or a sum that trace and fix have the same expressivity. Further recent papers in the area include $[1,8,32]$. 
An important algebraic connection made by the paper of Joyal et al. is revealed by a special case of their construction Int. Notice that a monoidal category in which the only arrows are identities is, ignoring the arrows, exactly a monoid structure on the objects, whereas a compact closed category with only identity arrows is an Abelian group. The notion of traced monoidal category in this special case turns out to be cancellative monoid. Again in this special case $\operatorname{Int}(\mathbf{C})$ is the universal Abelian group constructed from $\mathbf{C}$, which in the even more special case that $\mathbf{C}$ is the additive monoid of natural numbers yields $\operatorname{Int}(\mathbf{C})$ as the usual construction of (the additive group of) the integers.

The purpose of this paper is to show that there is more to this algebraic connection. A fundamental construction in the theory of commutative rings is the localization of a ring [26] relative to a multiplicative subset. Restricting to the multiplicative monoid this construction may be called the localization of monoid relative to a multiplicative subset. The construction may be made in two steps, first the construction of a monoid in which elements of the multiplicative set are cancellable (make $a$ in the monoid equivalent to $b$ if there is an $u$ in the multiplicative set such that $a u=b u$ ), followed by the Int construction, which provides multiplicative inverses for the elements of the multiplicative set. We will describe a categorical generalization of this process, but for simplicity restrict to the case where the multiplicative set is the whole monoid.

The categorical analogue of localization we have in mind is the universal construction of a compact closed category from a monoidal category. This construction can also be factored as a sequence of algebraic left adjoints:

$$
\text { MONOIDAL } \stackrel{\text { Circ }}{\longrightarrow} \text { FEEDBACK } \stackrel{Q}{\longrightarrow} \text { TRACED } \stackrel{\text { Int }}{\longrightarrow} \text { COMPACT. }
$$

Here $M O N O I D A L$ is the category of monoidal categories and strict monoidal functors. $F E E D B A C K$ is the category of monoidal categories with a trace-like feedback operation which however fails to satisfy two of the axioms of trace, one of the naturality axioms of trace and the yanking axiom (the failure of these axioms is due to delay in the feedback operation). Morphisms preserve tensor and feedback. $T R A C E D$ is the category of traced monoidal categories and trace preserving strict monoidal functors. COMPACT is the category of compact closed categories and functors preserving the compact closed structure. The first process in the sequence is the Circ construction of [18], the second step is a quotient $Q$ killing delay, and the third is the Joyal-Street-Verity Int construction. Notice that applying $\Pi_{0}$, the connected components functor, to a monoidal category yields a monoid, to a category with feedback or a traced monoidal category yields a cancellative monoid, and to a compact closed category yields an Abelian group. Under the effect of $\Pi_{0}$, Circ is the free cancellative monoid on a monoid, $Q$ is trivial, and Int is the free Abelian group on a cancellative monoid.

The Circ construction is defined as follows. From a monoidal category $\mathbf{C}$ form $\operatorname{Circ}(\mathbf{C})$ the monoidal category whose objects are the same as $\mathbf{C}$ and whose arrows from $A$ to $B$ (called circuits) are (isomorphism classes of) pairs $(\alpha, U)$ where $\alpha: A \otimes U \rightarrow B \otimes U$ is an arrow of $\mathbf{C}$; composition of $(\alpha, U)$ and $(\beta, V): B \rightarrow C$ 
is $\left(\pi_{1}(\beta \otimes U) \pi_{2}(\alpha \otimes V), U \otimes V\right)$ for suitable permutations $\pi_{1}, \pi_{2}$. In $[18,20]$ the authors described a wide range of examples of the Circ construction, including non-deterministic automata, Mealy automata, circuits, sequential algorithms, linear systems; an example not included there is the category of systems of recursive equations in an algebra. $\operatorname{Circ}(\mathbf{C})$ has a "feedback" operation, which in the examples is feedback with delay, and introduces new state to a system. There are precise delay elements $\delta_{A}=($ symmetry, $A): A \rightarrow A$.

The $C$ irc construction has its origins in the cascade product of automata in [25], and in the seminal paper of Elgot [11]. Variations of the construction have been used later by several authors including Bloom and Ésik [4], Stefanescu [33] and Bartha [2]. This last paper [2] is the most closely related to the present work. Bartha has a notion of strong feedback theory, a one-sorted algebraic theory with an operation of feedback with delay, a notion more special than our notion of category with feedback. A precise correspondence between the axioms is difficult because general feedback properties are mixed in [2] with properties special to the case that the tensor in a theory is a sum. In most of the examples of categories with feedback, or compact closed categories, of interest to us the tensor is not a product or a sum. To give one example, he has an axiom (S5) that feedback $\left(1_{X}: X \rightarrow\right.$ $X)=1_{I}: I \rightarrow I$; whereas in the classical example of finite dimensional spaces the trace of the identity function of a space $V$ is the dimension of $V$. The principal construction of his paper is the free strong feedback theory on a one-sorted theory. As a part of this construction Bartha defines $\operatorname{Circ}(\mathbf{T})$ for a one-sorted theory $\mathbf{T}$ (he calls it $A u t_{\mathbf{T}}$ ). The free strong feedback theory on $\mathbf{T}$ is then $A u t_{\overline{\mathbf{T}}} / \sim$ where $\overline{\mathbf{T}}$ is a modification of $\mathbf{T}$ and $\sim$ is a certain congruence. The definitions of $\overline{\mathbf{T}}$ and $\sim$ as given depend strongly on the fact that the theories are one-sorted.

Circuits have a variety of semantics. For example linear systems [20,28] have both discrete (unit-delay) and continuous (infinitesimal-delay) semantics, and in fact such delay semantics provide an important justification for the introduction of categories with delayed feedback as a separate concept. However in this paper we will discuss fixed point (zero-delay) semantics. We define a fixed point semantics for a category with feedback $\mathbf{D}$ to be a strong monoidal functor from $\mathbf{D}$ to a (not-necessarily-strict monoidal) compact closed category which takes feedback to trace. In the case that $\mathbf{D}$ is $\operatorname{Circ}(\mathbf{C})$ for some monoidal $\mathbf{C}$ this amounts to a strong monoidal functor from $\mathbf{C}$ to a compact closed category. A fixed point semantics kills the delay elements. Examples of fixed point semantics are (i) the (matrix of) languages recognized by a non-deterministic automaton, (ii) the partial function semantics of sequential programs (in the compact closed environment of Cospan(Sets)), (iii) the equilibrium behaviour of circuits (in compact closed Rel), and (iv) the set of fixed points of equations in an algebra (in compact closed Rel or Span).

Notice that we do not address the difficult question of the existence of fixedpoints which has been one of the central concerns of semantics, addressed for example in the work of Tarski and of Scott, though clearly there are connections between the trace semantics and the Lefschetz fixed point theorem. 
This paper is an extension of the first part of a talk [22] given by the third author at FICS 2001 (Firenze). The second part of that talk, which regarded the existence of solutions of fixed point equations in the cospan-span model of concurrency $[13,21]$ will be written up as a separate paper. Various results in this paper were announced in [20]. There categories with feedback were defined as algebras for a particular monad, while in this paper they are given a finite equational presentation.

\section{CAtegories With FEedBaCK}

As mentioned earlier, in this section of the paper monoidal category will mean strict symmetric monoidal category, and monoidal functor will mean strict monoidal functor. To simplify notation we will use the symbol $\pi$ as a generic symbol for a permutation in a monoidal category, where the particular permutation is clear from the context.

Remark 2.1. The definition of category with feedback is similar to part of the definition of trace monoidal category [15]; the yanking axiom is lacking, and one of the naturality axioms holds only in a weak form.

Definition 2.2. A category with feedback is a monoidal category $\mathbf{C}$ with for each triple of objects $A, B, U$ an operation

$$
f b k_{U}^{A, B}: \mathbf{C}(A \otimes U, B \otimes U) \rightarrow \mathbf{C}(A, B)
$$

satisfying the following axioms:

(i) (naturality)

$$
f b k_{U}\left((\beta: B \otimes U \rightarrow C \otimes U) \cdot\left((\alpha: A \rightarrow B) \otimes 1_{U}\right)\right)=f b k_{U}(\beta) \cdot \alpha
$$

(ii) (naturality)

$$
f b k_{U}\left(\left((\gamma: C \rightarrow D) \otimes 1_{U}\right) \cdot(\beta: B \otimes U \rightarrow C \otimes U)\right)=\gamma \cdot f b k_{U}(\beta)
$$

(iii) (weak naturality) If $\gamma: U \rightarrow V$ is an isomorphism in $\mathbf{C}$ then

$$
f b k_{U}\left((B \otimes \gamma) \cdot(\alpha: A \otimes U \rightarrow B \otimes U) \cdot\left(A \otimes \gamma^{-1}\right)\right)=f b k_{U}(\alpha)
$$

(iv) (vanishing)

$$
f b k_{I}(\alpha: A \rightarrow B)=\alpha
$$

(v) (vanishing)

$$
f b k_{U \otimes V}(\alpha: A \otimes U \otimes V \rightarrow B \otimes U \otimes V)=f b k_{U}\left(f b k_{V}(\alpha)\right)
$$


(vi) (superposing)

$$
f b k_{U}(\pi \cdot((\alpha: A \otimes U \rightarrow B \otimes U) \otimes(\beta: C \rightarrow D)) \cdot \pi)=f b k_{U}(\alpha) \otimes \beta
$$

Clearly any traced monoidal category, and hence any compact closed category, is a category with feedback.

Remark 2.3. The axioms also bear a close relation with axioms of Bloom and Ésik [5]. Axioms (i) and (ii) are analogues of the parameter identity. Axiom (iii) is the feedback permutation identity. Axiom (iv) is an analogue of a zero identity. Axiom (v) is an analogue of the pairing identity.

\subsection{The Circ construction}

Definition 2.4. Let $\mathbf{C}$ be a monoidal category. Then $\operatorname{Circ}(\mathbf{C})$ is the monoidal category defined as follows: objects of $\operatorname{Circ}(\mathbf{C})$ are those of $\mathbf{C}$; arrows from $A$ to $B$ are isomorphism classes of pairs $(\alpha, U)$ where $\alpha$ is an arrow in $\mathbf{C}$ from $A \otimes U$ to $B \otimes U$, and where an isomorphism from $(\alpha, U): A \rightarrow B$ to $(\beta, V): A \rightarrow B$ is an isomorphism $\gamma: U \rightarrow V$ in $\mathbf{C}$ such that $(B \otimes \gamma) \alpha=\beta(A \otimes \gamma)$. For simplicity, we will usually work in terms of representatives rather than equivalence classes of pairs. Tensor of objects is as in C. Identity, composition and tensor of arrows is as follows:

$$
\begin{gathered}
1_{A}=\left(1_{A}, I\right), \\
(\beta, V) \cdot(\alpha, U)=(\pi(\beta \otimes U) \pi(\alpha \otimes V), U \otimes V), \\
(\alpha, U) \otimes(\beta, V)=(\pi \cdot(\alpha \otimes \beta) \cdot \pi, U \otimes V) .
\end{gathered}
$$

Proposition 2.5. Circ $(\mathbf{C})$ is a category with feedback where the feedback operation is defined as follows:

$$
f b k_{U}((\alpha, V): A \otimes U \rightarrow B \otimes U)=(\alpha, U \otimes V) .
$$

Proof. Axiom(i)

$$
\begin{aligned}
f b k_{U}(((\beta, W) & \left.: B \otimes U \rightarrow C \otimes U) \cdot\left(((\alpha, V): A \rightarrow B) \otimes 1_{U}\right)\right) \\
& =(\pi \cdot(\beta \otimes V) \cdot \pi \cdot(\alpha \otimes U \otimes W) \cdot \pi, U \otimes V \otimes W): A \rightarrow C \\
& \simeq f b k_{U}(\beta, W) \cdot(\alpha, V) .
\end{aligned}
$$


Axiom(ii)

$$
\begin{aligned}
f b k_{U}((((\gamma, V) & \left.\left.: C \rightarrow D) \otimes 1_{U}\right) \cdot((\beta, W): B \otimes U \rightarrow C \otimes U)\right) \\
& =(\pi \cdot \gamma \otimes U \otimes W \cdot \pi \cdot \beta \otimes V, U \otimes W \otimes V): B \rightarrow D \\
& =(\gamma, V) \cdot f b k_{U}((\beta, W)) .
\end{aligned}
$$

Axiom(iii)

Suppose $(\gamma, P): U \rightarrow V$ is an isomorphism in $\operatorname{Circ}(\mathbf{C})$ with inverse $(\delta, Q)$. Then $(\gamma, P)(\delta, Q)=\left(\pi(\gamma \otimes Q) \pi(\delta \otimes P, Q \otimes P)=\left(1_{V}, I\right)\right.$. Hence in $\mathbf{C}$ the arrow $\pi \cdot(\gamma \otimes Q)$ : $U \rightarrow V$ is the inverse of $\pi \cdot(\delta \otimes P): V \rightarrow U$.

$$
\begin{aligned}
f b k_{U}((B \otimes(\gamma, P)) \cdot((\alpha, W) & : A \otimes U \rightarrow B \otimes U) \cdot(A \otimes(\delta, Q))) \\
& =\pi(B \otimes \gamma \otimes Q \otimes W) \pi(\alpha \otimes Q \otimes P) \pi(A \otimes \delta \otimes P \otimes W) \pi \\
& \cong f b k_{U}(\alpha) .
\end{aligned}
$$

Axiom(iv)

$$
f b k_{I}((\alpha, U): A \otimes I \rightarrow B \otimes I)=(\alpha, I \otimes U)=(\alpha, U): A \rightarrow B
$$

$\operatorname{Axiom}(\mathrm{v})$

$$
\begin{gathered}
f b k_{U \otimes V}((\alpha, W): A \otimes U \otimes V \rightarrow B \otimes U \otimes V)=(\alpha, U \otimes V \otimes W) \\
=f b k_{U}(\alpha, V \otimes W)=f b k_{U}\left(f b k_{V}(\alpha, W)\right) .
\end{gathered}
$$

$\operatorname{Axiom}(\mathrm{vi})$

$$
\begin{gathered}
f b k_{U}(\pi \cdot(((\alpha, V): A \otimes U \rightarrow B \otimes U) \otimes((\beta, W): C \rightarrow D)) \cdot \pi) \\
=\pi \cdot(\alpha \otimes \beta) \cdot \pi=f b k_{U}(\alpha) \otimes \beta .
\end{gathered}
$$

\subsection{Universality of the Circ COnstruction}

Notice that $\mathbf{C}$ is contained in $\operatorname{Circ}(\mathbf{C})$ via the functor $A \longmapsto A, \alpha \longmapsto(\alpha, I)$.

Proposition 2.6. Given any strict monoidal functor from $F: \mathbf{C} \rightarrow \mathbf{D}$ from a monoidal category to a category with feedback, there is a unique strict monoidal 
feedback-preserving functor

$$
G: \operatorname{Circ}(\mathbf{C}) \rightarrow \mathbf{D}
$$

extending $F$.

Proof. On objects $G$ is defined by $G(A)=F(A)$. On arrows $G((\alpha, U): A \rightarrow$ $B)=f b k_{F U}(F \alpha: F A \otimes F U \rightarrow F B \otimes F U)$. Noting that $(\alpha, U): A \rightarrow B=$ $f b k_{U}(\alpha: A \otimes U \rightarrow B \otimes U, I)$ in $\operatorname{Circ}(\mathbf{C})$, these definitions are clearly forced by the requirement that $G$ is feedback preserving and extends $F$.

First note that $G$ is well-defined on arrows. If $(\alpha, U) \simeq(\beta, V): A \rightarrow B$ then there is an isomorphism $\gamma: U \rightarrow V$ such that $(B \otimes \gamma) \cdot \alpha=\beta \cdot(A \otimes \gamma)$ or $(B \otimes \gamma) \cdot \alpha \cdot\left(A \otimes \gamma^{-1}\right)=\beta: A \otimes V \rightarrow B \otimes V$ in $\mathbf{C}$, so

$$
\begin{aligned}
G(\beta, V) & =f b k_{F V}(F \beta) \\
& =f b k_{F V}\left((F B \otimes F \gamma) \cdot F \alpha \cdot\left(F A \otimes F \gamma^{-1}\right)\right) \\
& =f b k_{F V}(F \alpha)=G(\alpha, U) .
\end{aligned}
$$

We need only to check that $G$ is a feedback-preserving strict monoidal functor.

(i) $G$ is a functor: consider $\alpha: A \otimes U \rightarrow B \otimes U, \beta: B \otimes V \rightarrow C \otimes V$. Then $(\beta, V)(\alpha, U)=(\pi(\beta \otimes U) \pi(\alpha \otimes V), U \otimes V)$, and hence

$$
\begin{array}{lr}
G((\beta, V)(\alpha, U)) & \\
=G((\pi(\beta \otimes U) \pi(\alpha \otimes V), U \otimes V)) & \text { (definition) } \\
=f b k_{F U \otimes F V}(\pi(F \beta \otimes F U) \pi(F \alpha \otimes F V)) & \text { (definition) } \\
=f b k_{F U}\left(f b k_{F V}(\pi(F \beta \otimes F U) \pi(F \alpha \otimes F V))\right) & \text { (vanishing) } \\
=f b k_{F U}\left(f b k_{F V}(\pi(F \beta \otimes F U) \pi)(F \alpha \otimes F V)\right) & \text { (naturality) } \\
=f b k_{F U}\left(\left(f b k_{F V}(F \beta) \otimes F U\right)(F \alpha \otimes F V)\right) & \text { (superposing) } \\
=f b k_{F V}(F \beta) f b k_{F U}(F \alpha) & \text { (naturality) } \\
=G((\beta, V)) G((\alpha, U)) . & \text { (definition) }
\end{array}
$$

(ii) $G$ is strict monoidal. Consider $\alpha: A \otimes U \rightarrow B \otimes U, \beta: C \otimes V \rightarrow D \otimes V$. Then

$$
(\alpha, U) \otimes(\beta, V)=\pi(\alpha \otimes \beta) \pi,
$$


and hence

$$
\begin{array}{lr}
G((\alpha, U) \otimes(\beta, V)) & \\
=G(\pi(\alpha \otimes \beta) \pi) & \text { (definition) } \\
=f b k_{F U \otimes F V}(\pi(F \alpha \otimes F \beta) \pi) & \text { (definition) } \\
=f b k_{F U}\left(f b k_{F V}(\pi(F \alpha \otimes F \beta) \pi)\right) & \text { (vanishing) } \\
=f b k_{F U}\left(\pi f b k_{F V}(F \alpha \otimes F \beta) \pi\right) & \text { (naturality) } \\
=f b k_{F U}\left(\pi\left(F \alpha \otimes f b k_{F V}(F \beta)\right) \pi\right) & \text { (superposing) } \\
=f b k_{F U}(F \alpha) \otimes f b k_{F V}(F \beta) & \text { (superposing) } \\
=G(\alpha, U) \otimes G(\beta, V) . & \text { (definition) }
\end{array}
$$

(iii) $G$ is feedback-preserving. Consider $\alpha: A \otimes U \otimes V \rightarrow B \otimes U \otimes V$. Then

$$
f b k_{U}((\alpha, V): A \otimes U \rightarrow B \otimes U)=(\alpha, U \otimes V),
$$

and hence

$$
\begin{aligned}
G\left(f b k_{U}((\alpha, V))\right. & =f b k_{F U \otimes F V}(F \alpha) & & \text { (definition) } \\
& =f b k_{F U}\left(f b k_{F V}(F \alpha)\right) & & \text { (vanishing) } \\
& =f b k_{F U}(G(\alpha)) . & & \text { (definition) }
\end{aligned}
$$

\subsection{TRACED MONOIDAL CATEGORIES FROM CATEGORIES WITH FEEDBACK}

Proposition 2.7. The free traced monoidal category on a category with feedback $\mathbf{D}$ is the category-with-feedback quotient $Q(\mathbf{D})$ of $\mathbf{D}$ obtained by adding the following axiom (yanking)

$$
f b k_{A}(\text { symmetry }: A \otimes A \rightarrow A \otimes A)=1_{A} .
$$

Proof. The only axiom of traced monoidal categories missing when yanking is added to categories with feedback is

(naturality)

$$
f b k_{U}\left(\left(1_{B} \otimes(\beta: V \rightarrow U)\right) \cdot(\alpha: A \otimes U \rightarrow B \otimes V)\right)=f b k_{V}\left(\alpha\left(1_{A} \otimes \beta\right)\right) .
$$

But Lemma 2.1 of [15] shows that naturality follows from weak naturality in the presence of the other axioms together with yanking.

It follows that the free compact closed category on a monoidal category $\mathbf{C}$ is $\operatorname{Int}(Q(\operatorname{Circ}(\mathbf{C}))$. 


\subsection{FIXED-POINT SEMANTICS}

Definition 2.8. A fixed-point semantics of a category with feedback $\mathbf{D}$ is a monoidal functor to a compact closed category, which takes feedback to trace.

Notice that if $\mathbf{D}$ is $\operatorname{Circ}(\mathbf{C})$ then a fixed point semantics of $\mathbf{D}$ is the same as a monoidal functor from $\mathbf{C}$ to a compact closed category.

\section{EXAMPLES}

All of the examples except the last are discussed in [20], though not explicitly their fixed point semantics in the sense of this paper. We sketch briefly the examples adding details not discussed in that paper. Throughout this section we relax our restriction that monoidal categories and functors are strict; clearly although we have only proved results in the strict case the results may be suitably generalized to non-necessarily-strict monoidal categories and strong monoidal functors.

We will use two compact closed categories as codomains for semantics. One is the category Rel [9], with objects sets, and arrows binary relations, tensor product being product of sets, and product of relations. The adjoint of $A$ is $A$; the unit $\eta: I \rightarrow A \times A$ is the relation $\{(*, a, a) ; a \in A\}$; the counit $\varepsilon: A \times A \rightarrow I$ is $\{(a, a, *) ; a \in A\}$. The other compact closed category is $\operatorname{Cospan}(\operatorname{Sets})[19]$, the category with objects sets, and arrows from $A$ to $B$ cospans $A \stackrel{f}{\longrightarrow} R \stackrel{g}{\longleftarrow} B$ of functions, with composition computed using pushouts. The tensor is binary coproduct + , and the unit $\eta: 0 \rightarrow A+A$ is the cospan $0 \stackrel{!}{\longrightarrow} A \stackrel{\nabla}{\longleftarrow} A+A$; the counit $\varepsilon: A+A \rightarrow 0$ is the copspan $A+A \stackrel{\nabla}{\longrightarrow} A \stackrel{!}{\longleftarrow} 0$.

\subsection{Deterministic Mealy automata}

Consider the monoidal category $($ Sets, $\times)$. A circuit from $A$ to $B$ in Sets, with product, is a pair $(\alpha, U)$ where $\alpha$ is a function $A \times U \rightarrow B \times U$. But this is the same thing as a deterministic Mealy automaton [31] with input alphabet $A$, output alphabet $B$, and state space $U$ (though lacking a specified initial state). Composition is essentially cascade product. One fixed point semantics is the functor Equilibrium : $\operatorname{Circ}($ Sets, $\times) \rightarrow \operatorname{Rel}$ defined by Equilibrium $(\alpha, U)=$ $\{(a, b): \exists u$ such that $\alpha(a, u)=(b, u)\}$. That is, Equilibrium $(\alpha, U)$ consists of those input/output pairs for which the system has an equilibrium state.

\subsection{Elgot automata}

Consider the monoidal category (Sets, + ). A circuit from $A$ to $B$ in Sets, with direct sum, is a pair $(\alpha, U)$ where $\alpha$ is a function $A+U \rightarrow B+U$ Such were called Elgot automata in $[17,35]$ and model sequential algorithms. (Notice that Rutten in [29] has used the name Elgot automaton for the different notion of a partial function $A \rightarrow A+B$.) The set $A$ may be thought of as initial states, the set $B$ as terminal states, and $U$ as internal states of an algorithm; then $\alpha$ is the 
next-state function. The usual semantics for such an automaton is the partial function $\phi: A \rightarrow B$ computed by $\alpha$ in the following sense: $\phi(a)$ is defined and equal to $b$ if there exists an $n$ such that for $0<i<n, \alpha^{i}(a)$ is defined and belongs to $U$ while $\alpha^{n}(a)=b \in B$. (In [30] it was shown that the recursive functions are precisely the semantics of Elgot automata constructible from the predecessor and successor functions using the operations of a distributive category.)

This usual semantics is not however an example of the fixed point semantics of this paper since the category of partial functions is not compact closed. A slight modification however does work. We take the compact closed domain of the semantics to be Cospan(Sets) and then a fixed point sematics for Elgot automata is:

$$
I / O:((\alpha, U): A \rightarrow B) \longmapsto \operatorname{trace}_{U, \text { Cospan }}\left(A+U \stackrel{\alpha}{\longleftrightarrow} B+U \stackrel{1_{B+U}}{\longleftarrow} B+U\right) .
$$

$I / O(\alpha, U)$ may be seen, by a simple calculation, to be $A \stackrel{\alpha \cdot i n j_{A}}{\longrightarrow}(B+U) / \sim \stackrel{i n j_{B}}{\longleftarrow} B$ where $u_{1} \sim u_{2}$ if $\alpha\left(u_{1}\right)=u_{2}$, and $u \sim b$ if $\alpha(u)=b$. That is, all the steps of the calculation are equated, but it is not the case that all non-terminating computations are equated. The usual partial function semantics is just the pullback of this pair of functions.

\subsection{Non-DETERMinistiC AUtOMATA}

Consider the monoidal category $\operatorname{Matr}\left(\wp\left(\Sigma^{*}\right)\right)$ with objects natural numbers, and arrows matrices of subsets of the free monoid $\Sigma^{*}$. Composition is matrix composition using the fact that $\wp\left(\Sigma^{*}\right)$ is a semiring, with union as addition and complex concatenation of sets of words as multiplication. Tensor product is + . In [7] non-deterministic automata on the alphabet $\Sigma$ with several initial and several terminal states were identified with certain arrows in $\operatorname{Circ}\left(\operatorname{Matr}\left(\wp\left(\Sigma^{*}\right)\right)\right)$. The category $\operatorname{Matr}\left(\wp\left(\Sigma^{*}\right)\right)$ is itself traced monoidal and hence the identity functor induces a semantics

$$
\operatorname{Circ}\left(\operatorname{Matr}\left(\wp\left(\Sigma^{*}\right)\right)\right) \rightarrow \operatorname{Matr}\left(\wp\left(\Sigma^{*}\right)\right) \rightarrow \operatorname{Int}\left(\operatorname{Matr}\left(\wp\left(\Sigma^{*}\right)\right)\right)
$$

A calculation shows that the semantics of an automaton is the matrix of languages recognized by the automaton.

\subsection{Systems of RECURSIVE EQUATIONS IN AN ALGEBRAIC THEORY}

Consider an equational theory $T$. For concreteness let us take the theory $T_{\text {Rings }}$ of commutative rings, though any theory, including an algebraic theory of computational processes would do. Objects are powers $R^{n}$ of a symbol $R$; arrows from $R^{m}$ to $R^{n}$ are $n$ tuples of polynomials in the $m$ variables $x_{1}, x_{2}, \ldots, x_{m}$. The tensor product is $\times$. Arrows of $\operatorname{Circ}\left(T_{\text {Rings }}\right)$ are pairs $\left(\alpha, R^{p}\right): R^{m} \times R^{p} \rightarrow R^{n} \times R^{p}$. Such an arrow consists of $n+p$ polynomials in $m+p$; denote the polynomials 
$f_{1}, f_{2}, \ldots, f_{n}, g_{1}, \ldots, g_{p}$. There is a corresponding system of recursive polynomial equations, which has the same content, namely,

$$
\begin{aligned}
y_{1} & =f_{1}\left(x_{1}, \ldots, x_{m}, u_{1}, \ldots, u_{p}\right) \\
y_{2} & =f_{2}\left(x_{1}, \ldots, x_{m}, u_{1}, \ldots, u_{p}\right) \\
& \ldots \\
y_{n} & =f_{n}\left(x_{1}, \ldots, x_{m}, u_{1}, \ldots, u_{p}\right) \\
u_{1} & =g_{1}\left(x_{1}, \ldots, x_{m}, u_{1}, \ldots, u_{p}\right) \\
& \ldots \\
u_{p} & =g_{p}\left(x_{1}, \ldots, x_{m}, u_{1}, \ldots, u_{p}\right) .
\end{aligned}
$$

From this point of view $\operatorname{Circ}\left(T_{\text {Rings }}\right)$ is an algebra of systems of equations, composition being substitution, feedback being the operation of equating a variable on the left with one on the right.

We will give, not one semantics, but one corresponding to each model of the theory, that is, to each ring $\mathbf{R}$. Following Lawvere, a ring $\mathbf{R}$ is a product-preserving functor from $T_{\text {Rings }}$ to Sets. The compact closed category we take is $(\mathbf{R e l}, \times)$. To give a semantics is to give a monoidal functor from $\left(T_{\text {Rings }}, \times\right)$ to $(\mathbf{R e l}, \times)$. We take that functor to be

$$
T_{\text {Rings }} \stackrel{\mathbf{R}}{\longrightarrow} \text { Sets } \stackrel{\text { inclusion }}{\longrightarrow} \text { Rel. }
$$

A calculation shows that the semantics of system (*) is the subset of $\mathbf{R}^{m} \times \mathbf{R}^{n}$ consisting of $m+n$ tuples $y_{1}, \ldots, y_{n}, x_{1}, \ldots, x_{m}$ of elements of the ring $\mathbf{R}$ such that there exist $u_{1}, \ldots, u_{p} \in \mathbf{R}$ satisfying the system of equations $(*)$. Note that an alternative semantics in $\mathbf{S p a n}(\mathbf{S e t s})$ instead of Rel would contain all the information of the solutions of the system, not just their existence.

Acknowledgements. The authors gratefully acknowledge stimulating conversations over many years with Stephen Bloom. We thank a referee for pointing out the work of Bartha. The research has been financially supported by the Dipartimento di Scienze Chimiche, Fisiche e Matematiche of the University of Insubria, Como, Italy, and by the Italian Progetto Cofinanziato MURST Tipi di Ordine Superiore e Concorrenza (TOSCA).

\section{REFERENCES}

[1] S. Abramsky, Retracing some paths in process algebras, Concur '96, edited by U. Montanari and V. Sassone. Springer-Verlag, Lecture Notes in Comput. Sci. 1119 (1996) 1-17

[2] M. Bartha, An algebraic model of synchronous systems. Inform. and Comput. 97 (1992) 97-131.

[3] L. Bernatsky and Z. Ésik, Sematics of flowchart programs and the free Conway theories. RAIRO: Theoret. Informatics Applic. 32 (1998) 35-78. 
[4] S.L. Bloom and Z. Ésik, Axiomatising schemes and their behaviours. J. Comput. System Sci. 31 (1985) 375-393.

[5] S.L. Bloom and Z. Ésik, Iteration Theories: The Equational Logic of Iterative Processes. Springer-Verlag, EATCS Monogr. Theoret. Comput. Sci. (1993).

[6] S.L. Bloom and Z. Ésik, Matrix and matricial iteration theories, Part I. J. Comput. System Sci. 46 (1993) 381-408.

[7] S.L. Bloom, N. Sabadini and R.F.C. Walters, Matrices, machines and behaviors. Appl. Categorical Structures 4 (1996) 343-360.

[8] R.F. Blute, J.R.B. Cockett and R.A.G. Seely, Feedback for linearly distributive categories: Traces and fixpoints. J. Pure Appl. Algebra 154 (2000) 27-69.

[9] A. Carboni and R.F.C. Walters, Cartesian Bicategories I. J. Pure Appl. Algebra 49 (1987) 11-32.

[10] J. Conway, Regular Algebra and Finite Machines. Chapman and Hall, London (1971).

[11] C.C. Elgot, Monadic computation and iterative algebraic theories, edited by J.C. Shepherdson. North Holland, Amsterdam, Logic Colloquium 1973, Studies in Logic 80 (1975).

[12] C.C. Elgot, Matricial Theories. J. Algebra 42 (1976) 391-421.

[13] F. Gadducci, U. Montanari, P. Katis, N. Sabadini and R.F.C. Walters, Comparing Cospanspans and Tiles via a Hoare-style process calculus. TOSCA Udine, Electron. Notes Theoret. Comput. Sci. 62 (2001) 152-171.

[14] M. Hasegawa, Models of Sharing Graphs: A categorical semantics of let and letrec, Ph.D. Thesis. Edinburgh (1997), Springer (1999).

[15] A. Joyal, R. Street and D. Verity, Traced monoidal categories. Math. Proc. Camb. Phil. Soc. 119 (1996) 447-468.

[16] A. Joyal and R. Street, Braided tensor categories. Adv. in Math. 102 (1993) 20-78.

[17] W. Khalil and R.F.C. Walters, An imperative language based on distributive categories II. RAIRO: Theoret. Informatics Appl. 27 (1993) 503-522.

[18] P. Katis, N. Sabadini and R.F.C. Walters, Bicategories of processes. J. Pure Appl. Algebra 115 (1997) 141-178.

[19] P. Katis, N. Sabadini and R.F.C. Walters, Span(Graph): A categorical algebra of transition systems, in Proc. Algebraic Methodology and Software Technology. Springer-Verlag, Lecture Notes in Comput. Sci. 1349 (1997) 307-321.

[20] P. Katis, N. Sabadini and R.F.C. Walters, On the algebra of systems with feedback and boundary. Rend. Circ. Mat. Palermo (2) Suppl. 63 (2000) 123-156.

[21] P. Katis, N. Sabadini and R.F.C. Walters, A formalization of the IWIM Model, in Proc. COORDINATION 2000, edited by A. Porto and G.-C. Roman. Springer-Verlag, Lecture Notes in Comput. Sci. 1906 (2000) 267-283.

[22] P. Katis, N. Sabadini and R.F.C. Walters, Recursion and concurrency, Invited talk, FICS 2001. Florence (2001).

[23] P. Katis and R.F.C. Walters, The compact closed bicategory of left adjoints. Math. Proc. Camb. Phil. Soc. 130 (2001) 77-87.

[24] G.M. Kelly and M. Laplaza, Coherence for compact closed categories. J. Pure Appl. Algebra 19 (1980) 193-213.

[25] K. Krohn and J. Rhodes, Algebraic theory of machines. I. Prime decomposition theorem for finite semigroups and machines. Trans. Amer. Math. Soc. 116 (1965) 450-464.

[26] M. Nagata, Local rings. Interscience (1962).

[27] R. Penrose, Applications of negative dimensional torsors, edited by D.J.A. Welsh. Academic Press, New York, Comb. Math. Appl. (1971) 221-244.

[28] W.J. Rugh, Linear System Theory, Second Edition. Prentice Hall (1996).

[29] J.J.M.M. Rutten, A calculus of transition systems (towards universal coalgebra), in Modal Logic and Process Algebra, a bisimulation perspective, edited by A. Ponse, M. de Rijke and Y. Venema. CSLI Publications, Standford, CSLI Lecture Notes 53 (1995) 231-256.

[30] N. Sabadini, S. Vigna and R.F.C. Walters, A note on recursive functions. Math. Struct. Comput. Sci. 6 (1996) 127-139. 
[31] M.W. Shields, An introduction to Automata Theory. Blackwell Scientic Publications, Oxford (1987).

[32] A. Simpson and G. Plotkin, Complete axioms for categorical fixed-point operators, in Proc. 15th LICS (2000) 30-41.

[33] Gh. Stefanescu, On flowchart theories I: The deterministic case. J. Comput. System Sci. 35 (1985) 163-191.

[34] G. Stefanescu, Network Algebra. Springer-Verlag (2000).

[35] R.F.C. Walters, Categories and Computer Science. Carslaw Publications (1991), Cambridge University Press (1992).

To access this journal online: www.edpsciences.org 